IOS Press

\title{
Preface
}

\section{Situation-aware intelligent environments}

\author{
Daniele Riboni ${ }^{\mathrm{a}}$ and Massimo Mecella ${ }^{\mathrm{b}}$ \\ ${ }^{a}$ Università di Cagliari, Italy \\ E-mail: riboni@unica.it \\ b Sapienza Università di Roma, Italy \\ E-mail:mecella@diag.uniromal.it
}

\section{This issue}

This Thematic Issue on "Situation-aware Intelligent Environments" of JAISE consists of five selected papers from the 14th International Conference on Intelligent Environments 2018, which was held in Rome (Italy) during June 25-28, 2018.

Intelligent Environments is one of the main components of the concept of Ambient Intelligence (AmI) and covers some key areas of research including, but not limited to, human activity recognition, humanenvironment interaction, and various machine learning and data mining techniques for applications such as smart home, healthcare, and smart cities. The emerging integration of objects able to sense, reason and communicate is enabling a new generation of services that assist the user in everyday tasks. For being effective, these services must be aware of the current situation, which includes not only location, but also current activity, mood, and social context, just to name a few. Objects in intelligent environments provide extensive low-level data that can be mined for capturing a fine-grained picture of the user's situation. Integrating and mining those data for situation-awareness is a challenging topic, which involves artificial intelligence, knowledge representation and reasoning, big data analysis, security, trust, and privacy issues.

The five papers appearing in this Thematic Issue cover a wide range of important topics for situationaware intelligent environments.

In smart manufacturing environments, wearable technologies and sensors bring the opportunity to use the recorded data to deliver timely assistance to the operator and to optimize work processes. However, the continuous acquisition of workers' sensor data determines relevant trust and privacy issues. The paper "A trust and privacy framework for smart manufacturing environments" by Mannhardt, Peterson, and Oliveira, proposes a framework to address trust and privacy challenges in complex smart manufacturing systems.

The rise of self-driving cars is paving the way to novel naturalistic interaction modalities, including feet gesture interaction. The paper "Enabling driver feet gestures using capacitive proximity sensing” by Frank and Kuijper, proposes and evaluates a technique to distinguish among four feet gestures; the technique does not cause privacy issues and has no visible interior design impact.

Heart disease and strokes are among the leading causes of death. Currently, electrocardiography monitoring is the only tool that helps physicians diagnose hearth issues. In the work "Utilising fog computing for developing a person-centric heart monitoring system" by Akrivopoulos, Amaxilatis, Mavrommati, and Chatzigiannakis, a novel system is proposed, which combines wearable embedded devices, mobile edge devices, and cloud services to provide reliable and accurate heart monitoring.

Today's low cost robots can perform their specific tasks, but they cannot cooperate to execute complex cooperative tasks. The paper "Tidy up my room: Multi-agent cooperation for service tasks in smart environments" by Rasch, Sprute, Pörtner, Battermann, and König, presents a system supported by cameras and lights to detect pointing gestures from a user and locating objects. The system in- 
creases the success rate of complex tasks execution by robots.

In environments with connected smart lighting, it is possible to deliver notifications using peripheral light, in order to avoid the intrusiveness of standard smartphone notification modalities such as sound, vibration and LEDs. The paper "Assessing the perceptibility of smartphone notifications in smart lighting spaces" by Komninos, Besharat, Stefanis, Gogoulou, and Garofalakis, investigates this issue by conducting experiments in a controlled environment that simulates a smart home environment. This study produces a set of guidelines for choosing notification modalities based on the current situation.

We hope that you will enjoy reading this Thematic Issue.

\section{Upcoming issues}

The following is the list of upcoming issues of JAISE:

- July 2019: Regular Issue

- September 2019: Thematic Issue on "Wearable Computing Techniques for Intelligent Health"

- November 2019: Regular Issue

- January 2020: Thematic Issue on "Cognitive Learning-based IoT Systems"'

More information on the call for papers for future thematic issues is available on the webpage of JAISE at: http://www.iospress.nl/journal/journal-ofambientintelligence-and-smart-environments/ 